\title{
How do stone attenuation and skin-to- stone distance in computed tomography influence the performance of shock wave lithotripsy in ureteral stone disease?
}

\author{
Gautier Müllhaupt, Daniel S. Engeler, Hans-Peter Schmid and Dominik Abt
}

\begin{abstract}
Background: Shock wave lithotripsy (SWL) is a noninvasive, safe, and efficient treatment option for ureteral stones. Depending on stone location and size, the overall stone-free rate (SFR) varies significantly. Failure of stone disintegration results in unnecessary exposure to shock waves and radiation and requires alternative treatment procedures, which increases medical costs. It is therefore important to identify predictors of treatment success or failure in patients who are potential candidates for SWL before treatment. Nowadays, noncontrast computed tomography (NCCT) provides reliable information on stone location, size, number, and total stone burden. The impact of additional information provided by NCCT, such as skin-to-stone distance (SSD) and mean attenuation value (MAV), on stone fragmentation in ureteral stone disease has hardly been investigated separately so far. Thus, the objective of this study was to assess the influence of stone attenuation, SSD and body mass index (BMI) on the outcome of SWL in ureteral stones.

Methods: We reviewed the medical records of 104 patients (80 men, 24 women) with ureteral stone disease treated consecutively at our institution with SWL between 2010 and 2013. MAV in Hounsfield Units (HU) and SSD were determined by analyzing noncontrast computed tomography images. Outcome of SWL was defined as successful (visible stone fragmentation on kidney, ureter, and bladder film (KUB)) or failed (absent fragmentation on KUB).
\end{abstract}

Results: Overall success of SWL was $50 \%$ (52 patients). Median stone attenuation was 956.9 HU (range 495-1210.8) in the group with successful disintegration and 944.6 (range 237-1302) in the patients who had absent or insufficient fragmentation. Median SSD was $125 \mathrm{~mm}$ (range $81-165 \mathrm{~mm}$ ) in the group treated successfully and $141 \mathrm{~mm}$ (range $108-172 \mathrm{~mm})$ in the patients with treatment failure. Unlike MAV $(p=0.37)$, SSD $(p<0.001)$ and BMI $(p=0.008)$ significantly correlated with treatment outcome.

Conclusion: The choice of treatment for ureteral stones should be based on stone location and size as considered in the AUA and EAU guidelines on urinary stone disease. In ambiguous cases, SSD and BMI can be used to assist in the decision. In this study, MAV showed no correlation with fragmentation rate of SWL.

Keywords: Ureteral stones, Treatment outcome, Shock wave lithotripsy, Hounsfield Units, Skin-to-stone distance

* Correspondence: gautier.muellhaupt@kssg.ch

Department of Urology, Cantonal Hospital St. Gallen, Rorschacherstrasse 95,

9007 St. Gallen, Switzerland 


\section{Background}

Shock wave lithotripsy (SWL) is a noninvasive, safe, and efficient treatment option for ureteral stones. Depending on stone location and size, the overall stone-free rate (SFR) varies significantly, leading to corresponding recommendations in the guidelines of the American Urological Association and the European Association of Urology: For proximal ureteral stones $<10 \mathrm{~mm}$, SWL has a higher SFR than ureterorenoscopy (URS), while URS seems to be superior for stones $>10 \mathrm{~mm}$. For mid-ureteral stones, URS appears to be superior, with statistical limitations, because fewer patients have been investigated. For distal stones $>10 \mathrm{~mm}$, URS is the treatment of choice, while SWL and URS are options for small stones [1, 2]. Failure of stone disintegration results in unnecessary exposure to shock waves and radiation, further patient suffering, and requires alternative treatment procedures, which increases medical costs [3]. It is therefore important to identify predictors of treatment success or failure in patients who are potential candidates for SWL before treatment.

Radiographic assessment of the stone is required to decide on the best treatment. Nowadays, noncontrast computed tomography (NCCT) provides reliable information on stone location, size, number, and total stone burden, and is therefore recommended as the standard diagnostic tool in urinary stone disease [3, 4]. Moreover, several studies have shown an impact of mean attenuation value (MAV) on treatment success of SWL in kidney stones, leading to corresponding guideline recommendations $[1,2]$. Despite the widespread use of NCCT, however, the impact of additional information provided by NCCT, such as skin-to-stone distance (SSD) and MAV, on stone fragmentation in ureteral stone disease has hardly been investigated separately so far [5-8]. Moreover, as limiting factors, three of the four studies reported on so far covered only one SWL session regardless of whether disintegration occurred or not, and treatment success was analyzed in all four studies at the earliest 2 weeks after SWL. The study by $\mathrm{Ng}$ et al. also included only proximal ureteral stones, and no real-time fluoroscopic screening was performed during treatment [7].

Thus, the objective of this study was to determine how additional information provided by NCCT and patient's physical constitution might influence fragmentation rate of SWL in ureteral stone disease.

Table 1 shows a summary of the literature.

\section{Methods}

One hundred four patients treated consecutively with SWL for distal and proximal ureteral stones in our department between January 2010 and December 2013 were included in this retrospective study. Data analysis was conducted according to the declaration of Helsinki and approved by the Local Ethics Committee of St. Gallen (EKSG 15/055). Written informed consent for data analysis was obtained. NCCT was performed before treatment using a multidetector row helical CT scanner (Siemens, Definition Flash, Forchheim, Germany) with 30-460 mA, $120 \mathrm{kV}$ and $2 \mathrm{~mm}$ collimation in every patient. As suggested in a study by Eisner et al. [9], stone size and Hounsfield Unit (HU) measurements were obtained in a standard bone window (window width-1,120 and window level-300). The image with the largest stone diameter was used to define maximum stone size. MAV was obtained by measuring the mean $\mathrm{HU}$ of defined regions of interest just smaller than the stone in magnified images without including adjacent soft tissue on each slice of the axial planes (Fig. 1). SSD was calculated as described by El Nahas et al. [3] and the distances at $0^{\circ}, 45^{\circ}$ and $90^{\circ}$ were measured using radiographic calipers (Fig. 2). The average was calculated as the SSD. The measurements were performed analogous in prone position when targeting pelvic stones. The SSD was also measured and evaluated at an angle of $90^{\circ}$ separately, as this seems to be the most important angle in the setting of the SLX-F2 (Storz Medical, Tägerwilen, Switzerland) which was used to perform SWL under sedoanalgesia.

If tolerated by the patient, up to 4,000 shocks (60-90/ min) with an energy level of up to 8 according to the manufacturer's scale were delivered during each SWL session. The energy level 8 corresponded to $16.4 \mathrm{kV}$ with the precise focus and $12.8 \mathrm{kV}$ with the extended focus. In patients with pain resistant to analgesic treatment, the energy and number of shocks were reduced according to the patient's tolerance. Stones were targeted and fragmentation was monitored by biplanar fluoroscopy at regular intervals during treatment.

Patients were further evaluated by kidney, ureter, and bladder (KUB) film, renal ultrasound, and sieving of urine to assess fragmentation, the presence of renal dilatation and expulsion of ureteral stones the day after the respective session. In cases of missing or inadequate disintegration in KUB, SWL was repeated once or twice at intervals of 1 day. The clinical outcome was defined as successful (visible stone fragmentation on KUB) or failed (absent fragmentation on KUB) immediately after the last SWL session.

The correlation with and influence of a range of baseline characteristics on treatment outcome of SWL was examined: patient's age, gender, weight, and BMI; stone location and volume, MAV, SSD; use of alpha blockers; presence of ureteral stents. Both univariate (chi-square or Mann-Whitney U-tests for dichotomous or continuous variables) and multivariate (binary logistic regression) analyses were performed to define significant factors. ROC curves were used for the determination of the best cut-off values. All tests were two-sided and a 
Table 1 Review of the literature

\begin{tabular}{|c|c|c|c|c|c|c|c|}
\hline \multirow[b]{2}{*}{ References } & \multirow[b]{2}{*}{ Year } & \multirow[b]{2}{*}{ Stone location } & \multirow[b]{2}{*}{ n All/Renal/Ureteral } & \multicolumn{3}{|l|}{ Prediction of successful disintegration/treatment } & \multirow[b]{2}{*}{ Cut off MAV/SSD/BMI } \\
\hline & & & & Mean attenuation value (MAV) All/Renal/Ureter & SSD All/Renal/Ureter & BMI All/Renal/Ureter & \\
\hline Joseph et al. [14] & 2002 & Renal & $30 / 30 /-$ & Yes/Yes/- & $-/-$ & $\mathrm{No} / \mathrm{No} /-$ & Renal: $950 \mathrm{HU} /-/-$ \\
\hline Pareek et al. [6] & 2003 & Renal and ureteral & $50 / 20 / 30$ & Yes/Yes/Yes & $-/-/$ & No/-/- & Ureteral: 900 HU/-/- \\
\hline Wang et al. [15] & 2005 & Renal & $80 / 80 /-$ & Yes/Yes/- & $-/-$ & $-/-/-$ & Renal: 900HU/-/- \\
\hline Gupta et al. [16] & 2005 & Renal and proximal ureter & 108/89/19 & Yes/-/- & $-/-/-$ & $-/-$ & All: 750 HU/-/- \\
\hline Yoshida et al. [17] & 2006 & Renal and proximal ureter & $56 / 25 / 31$ & Yes/-/- & $-/-/-$ & $-/-/-$ & $-/-/-$ \\
\hline El Nahas et al. [3] & 2007 & Renal & 120/120/- & Yes/Yes/- & Yes/Yes/- & Yes/Yes/- & Renal: $1000 \mathrm{HU} /-/-$ \\
\hline Perks et al. [11] & 2008 & Renal & 111/111/- & Yes/Yes/- & Yes/Yes/- & $\mathrm{No} / \mathrm{No} /-$ & Renal: $900 \mathrm{HU} / 9 \mathrm{~cm} /-$ \\
\hline Ng et al. [7] & 2009 & Proximal ureter & $94 /-194$ & Yes/-/Yes & Yes/-/Yes & $\mathrm{No} /-/ \mathrm{No}$ & Renal:593 HU/9.2 cm/- \\
\hline Patel et al. [10] & 2009 & Renal & 83/83/- & $\mathrm{No} / \mathrm{No} /-$ & Yes/Yes/- & $-/-/$ & Renal: $-/ 10 \mathrm{~cm} /-$ \\
\hline Wiesenthal et al. [5] & 2010 & Renal and ureteral & $422 / 218 / 204$ & Yes/Yes/Yes & Yes/Yes/Yes & Yes/No/Yes & All: 900 HU/ 11 cm/- \\
\hline Park et al. [12] & 2010 & Renal & 115/115/- & Yes/Yes/- & $\mathrm{No} /-/-$ & $-/-$ & Renal: 863 HU/-/- \\
\hline Shah et al. [18] & 2010 & Renal and proximal ureter & $99 / 71 / 28$ & Yes/-/- & $-/-/$ & $-/-/$ & $-/-/-$ \\
\hline Tanaka et al. [13] & 2013 & Renal and ureteral & $75 / 27 / 48$ & Yes/-/- & $\mathrm{No} /-/-$ & No/-/- & All: 780 HU/-/- \\
\hline Celik et al. [8] & 2015 & Renal and ureteral & 254/123/131 & Yes/Yes/Yes & -YYes/ & -/Yes/- & Renal: $750 \mathrm{HU} /-/-$ \\
\hline Nakasato et al. [19] & 2015 & Renal and ureteral & 260/92/168 & Yes/-/- & $\mathrm{No} /-/-$ & $-/-/$ & All: 815 HU/-/- \\
\hline
\end{tabular}




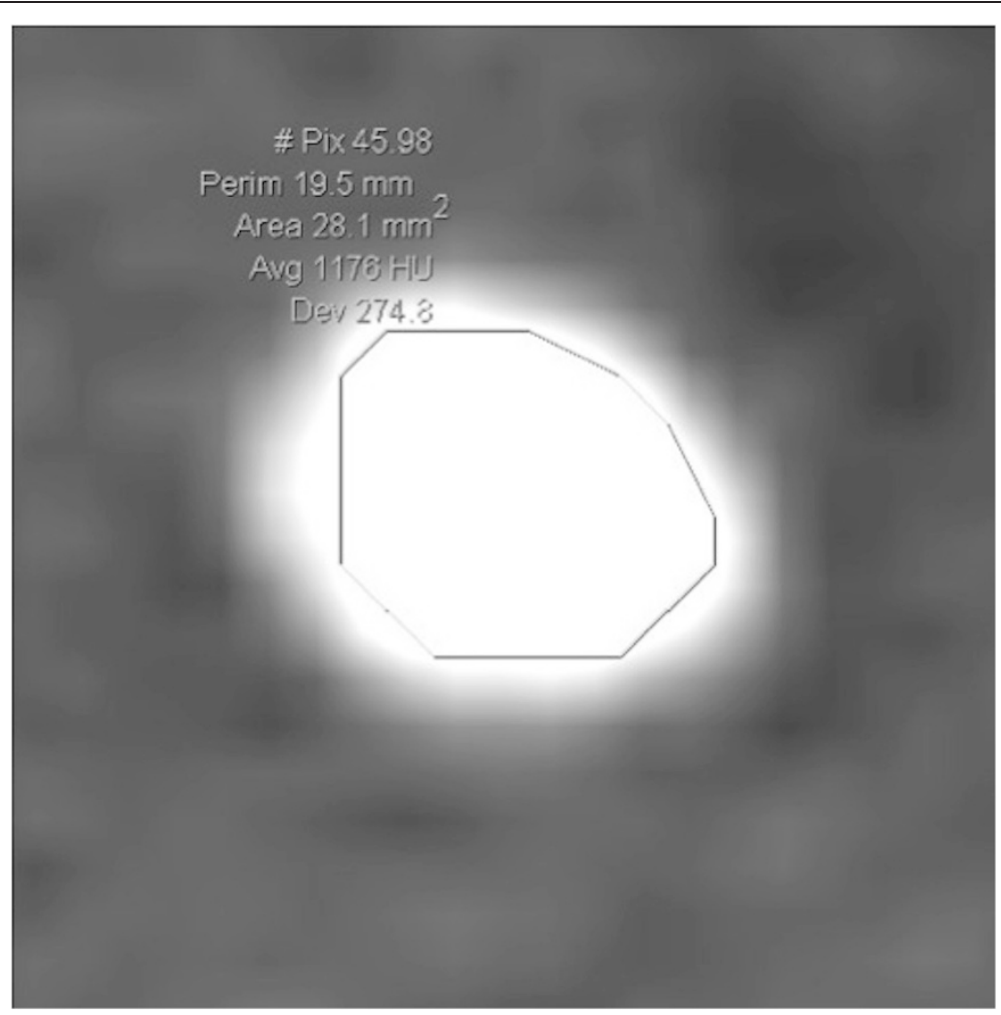

Fig. 1 Defined regions of interest just smaller than the stone without including adjacent soft tissue

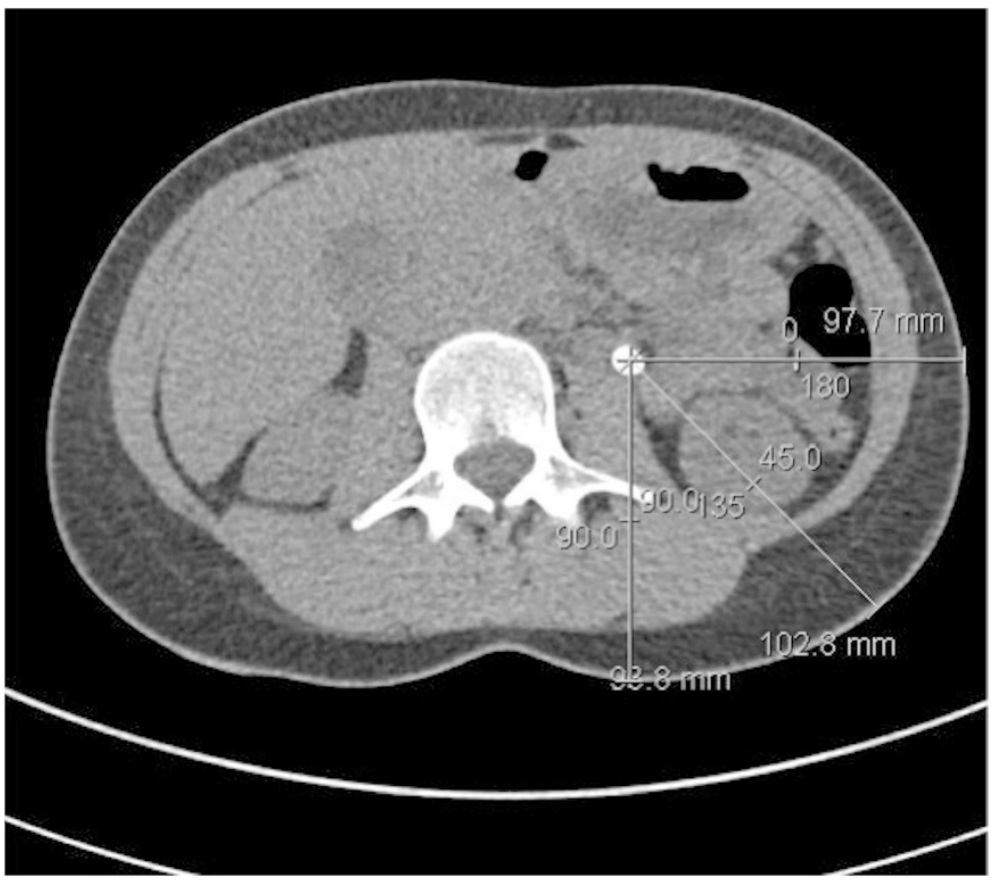

Fig. 2 Skin-to-stone distance (SSD) was calculated by the measuring distances from Stone-to-skin at $0^{\circ}, 45^{\circ}$, and $90^{\circ}$ using radiographic calipers 
p-value of $<0.05$ was necessary to reject the nullhypothesis. Statistical analyses were performed using IBM SPSS Statistics Version 22 (IBM Corp., New York, U.S.A.).

\section{Results}

A total of 104 consecutive patients were included (24 women, 80 men), median age was 45.5 years (range 19-80 years). Median largest diameter of stones was $6 \mathrm{~mm}$ (range 2-15 mm) and median MAV 949.3 HU (range 237-1302 $\mathrm{HU}$ ).

The stones were located in the proximal ureter in 73 (70.2 \%) patients and the distal ureter in 31 (29.8\%). Median BMI was 26.2 (range 17.4-37.0), median SSD $\left(0^{\circ} / 45^{\circ} / 90^{\circ}\right) 131.5 \mathrm{~mm}$ (range $81-172 \mathrm{~mm}$ ) and median $\operatorname{SSD}\left(90^{\circ}\right) 119 \mathrm{~mm}$ (range 76-161 mm).

Stone fragmentation was visible in $52(50 \%)$ patients and was not visible in the remaining patients, of whom 49 (94.2 \%) needed further treatment: $43(82.7 \%)$ by URS, 4 (7.7 \%) by ureteral stent insertion, and 2 (3.8 \%) by further cycles of SWL. The three patients who needed no further treatment showed spontaneous stone passage during the treatment with SWL without stone disintegration.

Of the 52 patients who showed good stone fragmentation, $13(25 \%)$ needed further treatment by URS ( 8 patients, $15.4 \%$ ), ureteral stent insertion (1 patient, $1.9 \%)$ or further cycles of SWL (4 patients, $7.7 \%$ ) because of impacted fragments or distal steinstrasse.
Median MAV was 956.9 HU (range 495-1210.8 HU) in patients with good stone fragmentation and was 944.6 $\mathrm{HU}$ (range 237-1302 HU) in patients showing no stone fragmentation and requiring further treatment. In univariate analysis, MAV showed no correlation with stone fragmentation $(p=0.373)$.

Median BMI, SSD $\left(0^{\circ} / 45^{\circ} / 90^{\circ}\right)$ and SSD $\left(90^{\circ}\right)$ in patients with good stone fragmentation were 25.5 (range 17.435.0), $125 \mathrm{~mm}$ (range 81-165 mm) and $114.5 \mathrm{~mm}$ (range 76-159 $\mathrm{mm}$ ). In patients without stone fragmentation, median BMI, SSD $\left(0^{\circ} / 45^{\circ} / 90^{\circ}\right)$ and $\operatorname{SSD}\left(90^{\circ}\right)$ were 27.1 (range 21.1-37.0), $141 \mathrm{~mm}$ (range 108-172 $\mathrm{mm}$ ) and 130 (range 85-161). In univariate analysis, BMI $(p=0.008)$, $\operatorname{SSD}\left(0^{\circ} / 45^{\circ} / 90^{\circ}\right) \quad(p<0.001)$ and $\operatorname{SSD}\left(90^{\circ}\right) \quad(p<0.001)$ significantly correlated with stone fragmentation.

In addition, maximum energy delivered showed a significant correlation with disintegration outcome $(p=0.015)$. Median energy level was 6 (range 4-8) in patients with good stone fragmentation and 6.4 (range 5-8) in patients with no stone fragmentation.

The results of univariate analyses are summarized in Table 2.

According to multivariate analyses, $\operatorname{SSD}\left(90^{\circ}\right)$ was a significant predictor for disintegration failure (regression coefficient: -0.046 , standard error: 0.013 , odds ratio $0.955,95 \%$ confidence interval: $0.930-0.980$, p-value < 0.001). Moreover, maximum delivered energy tended to be lower in patients with successful disintegration than

Table 2 Results of univariate analysis

\begin{tabular}{|c|c|c|c|}
\hline Characteristic & Successful disintegration & Unsuccessful disintegration & $p$-value \\
\hline Number of patients (\%) & $52(50 \%)$ & $52(50 \%)$ & - \\
\hline Age, years (median, range) & $43.5(19-80)$ & $47.5(22-77)$ & 0.136 \\
\hline Gender, M/F (N/\%) & $35(67.3 \%) / 17(32.7 \%)$ & $45(86.5 \%) / 7(13.5 \%)$ & 0.035 \\
\hline Weight, kg (median, range) & $73(49-116)$ & $85(58-120)$ & $<0.001$ \\
\hline $\mathrm{BMI}, \mathrm{kg} / \mathrm{m}^{2}$ (median, range) & $25.5(17.4-35.0)$ & $27.1(21.6-37.0)$ & 0.008 \\
\hline Skin-to-stone distance, $\mathrm{mm}$, mean of $0^{\circ}, 45^{\circ}$ and $90^{\circ}$ (median, range) & $125(81-165)$ & $141(108-172)$ & $<0.001$ \\
\hline Skin-to-stone distance, $\mathrm{mm}, 90^{\circ}$ (median, range) & $114.5(76-159)$ & $130(85-161)$ & $<0.001$ \\
\hline Mean attenuation value, HU (median, range) & $956.9(495-1210.8)$ & $944.6(237-1302)$ & 0.373 \\
\hline Stone size, mm (median, range) & $7(3-15)$ & $6(2-12)$ & 0.071 \\
\hline Location, proximal/distal (N, \%) & $36(69.2 \%) / 16(30.8 \%)$ & $37(71.2 \%) / 15(28.8 \%)$ & 1.000 \\
\hline SWL cycles (median, range) & $2(1-3)$ & $2(1-3)$ & 0.786 \\
\hline Number of shockwaves (median, range) & $8000(1000-12000)$ & $8000(3000-14000)$ & 0.583 \\
\hline Power/Intensity Level (median, range) & $6(4-8)$ & $6.4(5-8)$ & 0.015 \\
\hline Ureteral stent in place $(\mathrm{N}, \%)$ & 15 (28.8\%) & $13(25 \%)$ & 0.825 \\
\hline Alpha-blocker (N, \%) & $42(80.8 \%)$ & $38(73.1 \%)$ & 0.486 \\
\hline \multicolumn{4}{|l|}{ Secondary procedures } \\
\hline URS (N, \%) & $8(15.4 \%)$ & $43(82.7 \%)$ & - \\
\hline Ureteral stent (N, \%) & $1(1.9 \%)$ & $4(7.7 \%)$ & - \\
\hline SWL (N, \%) & $4(7.7 \%)$ & $2(3.8 \%)$ & - \\
\hline
\end{tabular}


in patients with disintegration failure without reaching statistical significance (regression coefficient: -0.528 , standard error: 0.272 , odds ratio $0.590,95 \%$ confidence interval: 0.346-1.004, p-value $<0.052$ ). Weight and BMI of the patient were not included in the multivariate analysis because of multicollinearity with SSD.

The ROC curves for different parameters were analyzed to find the optimum cut-off values to predict disintegration failure (Fig. 3). The optimum cut-off point for SSD $\left(90^{\circ}\right)$ would be $>11.9 \mathrm{~cm}$ (sensitivity $65.4 \%$, specificity $65.3 \%$ ), for patient weight $>82.5 \mathrm{~kg}$ (sensitivity $65.4 \%$, specificity $71.4 \%$ ), and for BMI $>25.9 \mathrm{~kg} / \mathrm{m}^{2}$ (sensitivity $69.2 \%$, specifity $55.1 \%$ ).

\section{Discussion}

The results of this study show that SSD and BMI are significant predictors of the outcome of SWL. As described earlier by Patel et al. for kidney stones [10], we found no significant association between MAV and fragmentation rate of SWL for ureteral stones.

The use of NCCT for diagnosis of ureteral stones is well established and a common practice worldwide [7]. The method of measuring SSD in NCCT has been well described in the literature and there are only marginal differences between studies with regard to the method [3, 5-7, 10-13].

The method for determining MAV has been described inconsistently, however. For example, Joseph et al. [14] used a calculous pixel map of 100 attenuation values in a $10 \times 10$ matrix in unenhanced axial NCCT section, while Wiesenthal et al. [5] measured attenuation values using bone windows on the magnified, axial image of the stone in the maximum diameter where the elliptical region of interest incorporated the largest cross-sectional area of stone without including adjacent soft tissue. In our study, we determined MAV by measuring the mean $\mathrm{HU}$ of defined regions of interest just smaller than the stone in magnified images without including adjacent soft tissue on each slice of the axial planes (Fig. 1) with a standard bone window (window width-1,120 and window level-300) as suggested in the study by Eisner et al. [9]. We believe that this is the most accurate method of determining MAV. The inconsistent methods used in the literature might also explain the differing results that have been reported, so far (Table 2). In our opinion, image magnification for MAV measurement is very important because accurate stone margins can be identified using only adequately magnified images (Fig. 1). Thus, inclusion of adjacent soft tissue into measurement can be avoided. In addition, we measured all available slices of stones in axial planes to calculate MAV, which might prevent assumption of too high or low MAVs, as stones often consist of different components. The method of measuring MAV should be standardized to allow comparison of different datasets.

Concerning MAV, cut-off values between 750 and 1000 HU for renal calculi and between 750 and $900 \mathrm{HU}$ in studies examining mixed ureteral and renal stones have

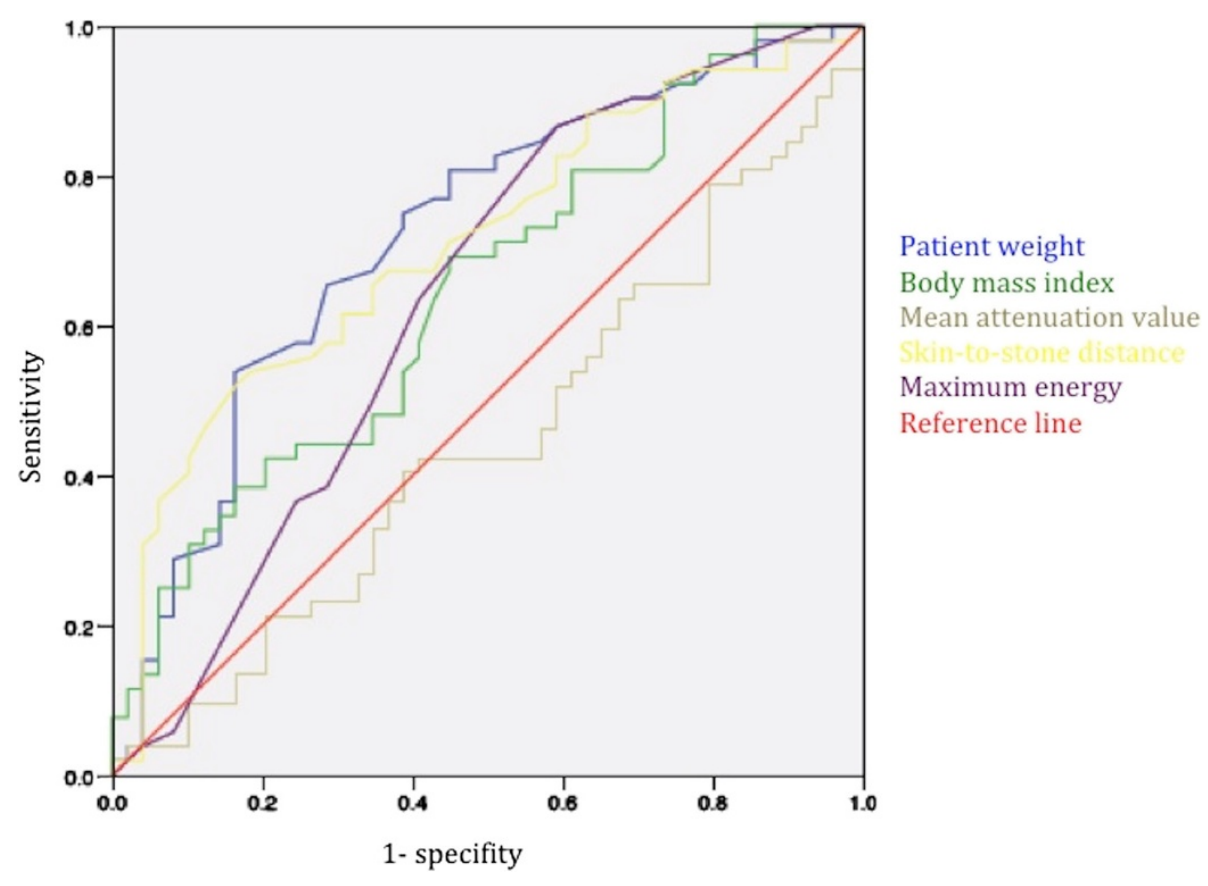

Fig. 3 ROC curves 
been suggested as predictors of SWL failure (Table 2). However, separate examination of cut-off values for ureteral stones has only been performed in two studies: Pareek et al. [6] suggested $900 \mathrm{HU}$ as the cut-off value in their study of 30 ureteral stones, and $\mathrm{Ng}$ et al. [7] defined a very different threshold of $593 \mathrm{HU}$ as a potential predictor of treatment success in a study in 94 patients with upper ureteral stones. Our study failed to show an association between MAV and the disintegration of ureteral stones using SWL.

SSD has been shown to be a significant predictor of the outcome of SWL in different studies on renal stones. The findings for BMI, however, have been inconsistent as illustrated in Table 2.

Only two studies have analyzed SSD as a predictor of SWL treatment success in ureteral stones separately $[5,7]$ and both showed that SSD was a significant predictor. In this context, $\mathrm{Ng}$ et al. [7] suggested an SSD cut-off of $9.2 \mathrm{~cm}$ as a predictor for SWL failure, but they studied only upper ureteral stones.

In our study, $\operatorname{SSD}\left(90^{\circ}\right)$ emerged as an even stronger predictor of treatment success or failure than mean SSD, with a cut-off value of $11.9 \mathrm{~cm}$ for SWL failure, which might be because we had patients in the almost straight supine or prone position for treatment with SLX-F2.

Results for BMI as a predictor of stone disintegration with SWL are also contradictory [5, 7]. In our study, BMI and patient weight were significant predictors of SWL outcome with cut-off values of $25.9 \mathrm{~kg} / \mathrm{m}^{2}$ and $82.5 \mathrm{~kg}$ for SWL failure. Factors such as differences in body fat distribution between men and women, and age and race also have to be taken into consideration.

Possible limitations of our study are the retrospective design and assessment of disintegration outcome by KUB film and not by NCCT. Moreover, fragmentation rate instead of SFR was chosen to define treatment success or failure. We believed that this might represent the stone's response to SWL better, because SFR is influenced by other factors that might interfere with stone passage (e.g., ureteral diameter). On the other hand, stone disintegration on KUB does not inevitably lead to a successfully completed treatment.

\section{Conclusions}

The choice of treatment for ureteral stones should be based on stone location and size as considered in the AUA and EAU guidelines on urinary stone disease. Patient preference also has to be taken into consideration. In ambiguous cases, SSD and BMI - in contrast to MAV - can be easily used for additional guidance. In this way, patients with a high risk of disintegration failure could be educated more precisely, unnecessary exposure to shock waves and radiation could be avoided and medical costs could be reduced.

\section{Abbrevations}

SSD: Skin-to-stone distance; MAV: Mean attenuation value; HU: Hounsfield Units; SWL: Shock wave lithotripsy; BMI: Body mass index; AUA: American Urological Association; EAU: European Association of Urology; SFR: Stone-free rate; URS: Ureterorenoscopy; NCCT: Noncontrast computed tomography; CT: Computed tomography; kV: Kilovolt; KUB: Kidney, ureter, and bladder film; ROC: Receiver operating characteristic.

\section{Competing interests}

The authors declare that they have no competing interests.

\section{Authors' contributions}

GM: Conception, study design, acquisition of data, analysis and interpretation of data, manuscript drafting. DE: Analysis and interpretation of data, statistics, proofreading. HPS: Analysis and interpretation of data, proofreading. DA:

Conception, study design, analysis and interpretation of data, proofreading. All authors read and approved the final manuscript.

\section{Acknowledgments}

The authors thank Alistair Reeves for editing the manuscript. No funding has been obtained for this study.

Received: 6 January 2015 Accepted: 20 July 2015

Published online: 23 July 2015

\section{References}

1. Preminger GM, Tiselius HG, Assimos DG, Alken P, Buck C, Gallucci M, et al. 2007 Guideline for the management of ureteral calculi. J Urol. 2007;178:2418

2. Preminger GM, Tiselius HG, Assimos DG, Alken P, Buck C, Gallucci M, et al. 2007 Guideline for the management of ureteral calculi. Eur Urol. 2007:52:1610.

3. El-Nahas AR, El-Assmy AM, Mansour O, Sheir KZ. A prospective multivariate analysis of factors predicting stone disintegration by extracorporeal shock wave lithotripsy: the value of high-resolution noncontrast computed tomography. Eur Urol. 2007:51:1688.

4. Williams Jr JC, Kim SC, Zarse CA, McAteer JA, Lingeman JE. Progress in the use of helical CT for imaging urinary calculi. J Endourol. 2004;18:937.

5. Wiesenthal JD, Ghiculete D, D'A Honey RJ, Pace KT. Evaluating the importance of mean stone density and skin-to-stone distance in predicting successful shock wave lithotripsy of renal and ureteric calculi. Urol Res. 2010;38:307

6. Pareek G, Armenakas NA, Fracchia JA. Hounsfield units on computerized tomography predict stone-free rates after extracorporeal shock wave lithotripsy. J Urol. 2003;169:1679.

7. Ng CF, Siu DY, Wong A, Goggins W, Chan ES, Wong KT. Development of a scoring system from noncontrast computerized tomography measurements to improve the selection of upper ureteral stone for extracorporeal shock wave lithotripsy. J Urol. 2009;181:1151.

8. Celik S, Bozkurt O, Kaya FG, Egriboyun S, Demir O, Secil M, et al. Evaluation of computed tomography findings for success prediction after extracorporeal shock wave lithotripsy for urinary tract stone disease. Int Urol Nephrol. 2015;47:69.

9. Eisner BH, Kambadakone A, Monga M, Anderson JK, Thoreson AA, Lee $H_{\text {, }}$ et al. Computerized tomography magnified bone windows are superior to standard soft tissue windows for accurate measurement of stone size: an in vitro and clinical study. J Urol. 2009;181:1710.

10. Patel T, Kozakowski K, Hruby G, Gupta M. Skin to stone distance is an independent predictor of stone-free status following shockwave lithotripsy. J Endourol. 2009:23:1383.

11. Perks AE, Schuler TD, Lee J, Ghiculete D, Chung DG, D'A Honey RJ, et al. Stone attenuation and skin-to-stone distance on computed tomography predicts for stone fragmentation by shock wave lithotripsy. Urology. 2008;72:765.

12. Park $\mathrm{Yl}, \mathrm{Yu} \mathrm{JH}$, Sung $\mathrm{LH}$, Noh $\mathrm{CH}$, Chung JY. Evaluation of possible predictive variables for the outcome of shock wave lithotripsy of renal stones. Korean J Urol. 2010;51:713.

13. Tanaka M, Yokota E, Toyonaga Y, Shimizu F, Ishii Y, Fujime M, et al. Stone attenuation value and cross-sectional area on computed tomography predict the success of shock wave lithotripsy. Korean J Urol. 2013;54:454. 
14. Joseph P, Mandal AK, Singh SK, Mandal P, Sankhwar SN, Sharma SK. Computerized tomography attenuation value of renal calculus: can it predict successful fragmentation of the calculus by extracorporeal shock wave lithotripsy? A preliminary study. J Urol. 2002;167:1968.

15. Wang LJ, Wong YC, Chuang CK, Chu SH, Chen CS, See LC, et al. Predictions of outcomes of renal stones after extracorporeal shock wave lithotripsy from stone characteristics determined by unenhanced helical computed tomography: a multivariate analysis. Eur Radiol. 2005;15:2238.

16. Gupta NP, Ansari MS, Kesarvani P, Kapoor A, Mukhopadhyay S. Role of computed tomography with no contrast medium enhancement in predicting the outcome of extracorporeal shock wave lithotripsy for urinary calculi. BJU Int. 2005;95:1285.

17. Yoshida S, Hayashi T, Ikeda J, Yoshinaga A, Ohno R, Ishii N, et al. Role of volume and attenuation value histogram of urinary stone on noncontrast helical computed tomography as predictor of fragility by extracorporeal shock wave lithotripsy. Urology. 2006;68:33.

18. Shah K, Kurien A, Mishra S, Ganpule A, Muthu V, Sabnis RB, et al. Predicting effectiveness of extracorporeal shockwave lithotripsy by stone attenuation value. J Endourol. 2010;24:1169.

19. Nakasato T, Morita J, Ogawa Y. Evaluation of Hounsfield Units as a predictive factor for the outcome of extracorporeal shock wave lithotripsy and stone composition. Urolithiasis. 2015;43:69.

\section{Submit your next manuscript to BioMed Central and take full advantage of:}

- Convenient online submission

- Thorough peer review

- No space constraints or color figure charges

- Immediate publication on acceptance

- Inclusion in PubMed, CAS, Scopus and Google Scholar

- Research which is freely available for redistribution 